\title{
The epidemiology and clinical manifestation of neurological melioidosis: A series of 19 cases from Southwest India over 10 years
}

\author{
I Halim ${ }^{1}$, T Shaw ${ }^{1}$, TAK Chaitanya ${ }^{1}$, KE Vandana $^{1}$, M Hande $^{2}$ GMenon $^{3}$, SP Gorthy ${ }^{3}$, \\ C Mukhopadhyay ${ }^{1}$
}

\section{Introduction}

Melioidosis, a syndrome with protean clinical manifestations, is caused by a Gram negative soil saprophyte, Burkholderiapseudomallei. Among its diverse clinical presentations, pulmonary, cutaneous, osseous, deep-organ, and septicemic foci are well-known. Neurological manifestations are however, less frequently observed and the microbiological evidence of such is even more rare. This study was undertaken to obtain newer insights into the clinical spectrum of neurological melioidosis, including its presentation, microbiological and radiological diagnosis and associated risk factors.

\section{Methods}

Neurological manifestations in patients diagnosed with culture confirmed melioidosis were documented during the period of January 2006 to December 2016. Basic demographics and risk factors associated with neurological complications were determined.

\section{Results}

Among 200 patients with melioidosis, 19 (9.5\%) presented with neurological manifestations. The mean age was $46.8 \pm 13.9$ years (range 7-67 years). The majority of the patients were male $(16 / 19 ; 84.2 \%)$. Most presented during the monsoon (14/19 73.6\%). Common clinical presentations observed were fever $(16 ; 84.2 \%)$, headache $(63.5 \%)$, paresthesia $(47.3 \%)$ and lower limb paresis (36.8\%). Magnetic resonance imaging (MRI) showed that brain abscess was the most common finding. Diabetes mellitus was the most common risk factor (68.4\%). Blood culture had more diagnostic value than CSF culture in patients with neurological melioidosis. Overall mortality was $31.5 \%$ (6/19).

\section{Discussion and Conclusions}

Neurological signs and symptoms have not been classically associated with melioidosis. Treating physicians should consult the clinical microbiologist whenever they have any suspicion of such cases and the microbiology laboratory should be well-equipped to diagnose melioidosis from CSF and/or blood samples.

\footnotetext{
${ }^{I}$ Department of Microbiology, Kasturba Medical College and Hospital, Manipal, Karnataka, India

${ }^{2}$ Department of Medicine, Kasturba Medical College and Hospital, Manipal, Karnataka, India ${ }^{3}$ Department of Neurosciences, Kasturba Medical College and Hospital, Manipal, Karnataka, India

Address for correspondence: Dr IsraHalim, Department of Microbiology, Kasturba Medical College and Hospital, Manipal, Karnataka, India.+7760532340 Email: israhalim@gmail.com (Dttps://orcid.org/0000-0002-8890-237X
} 